\title{
Hommage à André GUINIER, Membre de 1'Institut
}

André Guinier fait partie de ces grandes figures dont on aime se remémorer régulièrement des étapes de leur vie, celles qui nous ont le plus marqués, et découvrir au travers de discussions certains aspects dont nous n'avions pas connaissance. En outre il ne paraît pas inutile de retracer, pour les jeunes en particulier, mais aussi simplement par hommage, certains aspects des avancées qui leur sont dues. Ils ont forcé notre admiration et nous aimons en raviver régulièrement le souvenir.

André Guinier s'est éteint le 3 juillet 2000. II allait avoir 89 ans. II était né, en effet, le $1^{\text {er }}$ août 1911 à Nancy. Après de brillantes études dans cette ville, il entre à l'École Normale Supérieure en 1935. II disait d'ailleurs qu'il n'avait jamais imaginé faire autre chose. II prépare ensuite sa thèse à partir de 1936. II est alors assistant, et demande à Charles Mauguin (un cristallographe connu, auquel on doit en particulier la notation des systèmes de symétries que beaucoup d'entre nous utlisent) d'être son " patron de thèse ". II la soutient en 1939. C'est Mauguin qui lui propose de monter une chambre de rayons X avec un faisccau primaire monochromatique. Cet appareil, qui devint la chambre de Guinier, fut terminé au bout d'une année de travail. II devenait alors possible de mesurer la diffusion des rayons $X$ à petit angle car cette chambre n'avait pas d'effets parasites à petit angle, à l'inverse de celles utilisées jusque là par les autres chercheurs. André Guinier, qui était avant tout un expérimentateur de très grand talent, avait alors noté qu'aucun signal à petit angle n'était observé avec un échantillon homogène, mais qu'un matériau à petits grains (quelques dizaines de nanomètres) donnait de l'information dans ces domaines des petits angles. II devint alors capable de déterminer la distribution de taille des grains à partir de la courbe de diffusion angulaire. C'est pour ces raisons expérimentales que put alors se développer cette technique de diffusion à petits angles, pour les rayons $X$ ou les neutrons, qui est devenue si utile en métallurgie et plus généralement en physique des solides dans l'étude des matériaux.

André Guinier m'avait poussé à organiser avec lui, il y a une vingtaine d'années, une série d'entretiens faisant intervenir des personnalités scientifiques. L'idée était de laisser des témoignages directs sur l'évolution ou l'apparition de domaines comme la métallurgie, la physique des solides, la microscopie électronique... Au cours de l'une de ces discussions, cette fois-là avec Jacques Friedel, le 2 mars 1999, André Guinier nous a raconté l'histoire de la découverte des amas qui portent son nom. Cela se passe en 1938, il recherche pour le montage d'un appareil de diffraction des rayons $X$, une fenêtre mince en métal léger. On lui suggère d'aller voir J. Calvet qui travaille au Collège de France avec P. Jacquet, et chez qui il pourra sans doute trouver ce qu'il désire. Avant de quitter " la petite pièce où se trouve Calvet » avec la précieuse fenêtre, il profite de cette visite pour lui demander quel est l'objet de ses recherches. Celui-ci explique alors qu'il travaille sur les alliages légers. Lorsqu'on met du cuivre dans de l'aluminium, l'alliage devient plus dur après trempe suivie d'un léger recuit. On appelle cela le durcissement structural. C'était connu depuis les travaux de Wilm au début du siècle (1911), mais personne ne comprend alors son origine. On dit que des atomes de cuivre doivent se rassembler en amas, mais personne n'a d'idée précise. Cela se passait à $11 \mathrm{~h}$ du matin. " Donnez-moi un échantillon, moi je peux vous dire ce qui se passe et où sont les atomes ". À quatre heures de l'après-midi, ayant mis l'échantillon dans son appareil et développé les plaques exposées, il était devenu clair pour André Guinier que le cuivre se rassemblait dans les plans 100 sous forme d'amas d'une épaisseur d'un plan atomique. Un Compte-Rendu de l'Académie des Sciences paraît aussitôt. C'était donc en 1938. Tout cela avait pu être réalisé parce qu'André Guinier était devenu un grand et fin connaisseur de la radiocristallographie X. II dit dans cet entretien " Je ne connaissais rien au durcissement structural "! Ce fut pourtant le grand début. Et il devait apprendre très rapidement ce qu'était un alliage et plus généralement un matériau, et devenir un très grand spécialiste de ce domaine.

Ce qui est également étonnant, c'est que la même découverte est faite indépendamment cette même année, quelques semaines plus tard, par G.D. Preston qui, lui, travaillait sur la structure des alliages depuis quelques années. La démarche était donc tout à fait différente. D'un côté c'est la mise au point d'une technique très fine et une rencontre fortuite qui vont entraîner une découverte majeure. De l'autre côté on trouve un métallurgiste qui recherchait depuis longtemps la raison du comportement d'un alliage et qui aboutit au même résultat. André Guinier disait souvent qu'il avait eu beaucoup de chance et que celle-ci était souvent présente en recherche.

En 1944, André Guinier obtient le poste de chef de service puis de directeur-adjoint, du Laboratoire d'essais du Conservatoire National des Arts et Métiers. II devient très vite (1946) collaborateur scientifique de l'ONERA, fonction qu'il assumera pendant onze années. C'est durant cette période, juste après la deuxième guerre mondiale, que Raimond Castaing vient le voir au CNAM. Ils aimaient rappeler tous les deux leur première rencontre dans la cour du CNAM. André Guinier lui propose alors un sujet de thèse. II s'agit d'utiliser les rayons X caractéristiques (séparés en longueur d'onde par un cristal) émis sous l'impact d'une sonde d'électrons afin de réaliser l'analyse élémentaire de l'objet étudié. Raimond Castaing réalise alors la première microsonde. L'analyse élémentaire locale, la microanalyse est inventée. Les premiers résultats sortent en 1948 et la première publication est cosignée par Castaing et Guinier. La thèse de Castaing, qui reste le pilier de la microanalyse, est soutenue en 1951.

En 1949 André Guinier devient Maître de Conférences à la Faculté des Sciences de Paris. II y est ensuite nommé Professeur titulaire (1954). Et c'est en 1959 qu'il devient titulaire de la chaire de Physique des Solides à la Faculté des Sciences de Paris-Orsay. 
Les cours d'André Guinier, au CNAM, portaient en particulier sur la radiocristallographie. À la Sorbonne, son cours d'électromagnétisme dans "l'amphi aux vaches » nous a laissé un souvenir encore très présent. Un jour, après une belle démonstration de la formule de Rayleigh, il a terminé son cours en nous déclarant «... et c'est pour cela que le ciel est bleu ». Cette démonstration devait beaucoup lui plaire puisqu'une quarantaine d'années après, il a écrit « La structure de la matière, du ciel bleu à la matière plastique ».

En 1958, Jacques Friedel, Raimond Castaing et André Guinier se réunissent sous l'impulsion de ce dernier pour fonder ce qui va devenir le Laboratoire de Physique des Solides d'Orsay. Cette idée se concrétise vraiment en 1959. Oser aller à Orsay, loin de la capitale, n'était pas banal. Cette démarche était même un peu iconoclaste. Mais il a su convaincre Jacques Friedel et Raimond Castaing et bien d'autres, dont les autorités administratives, d'y installer ce laboratoire commun. Ils proposent peu après à André Jacquinot, qui était alors directeur général du CNRS, de rassembler officiellement leurs forces pour fonder ce qui allait devenir un laboratoire associé au CNRS, le LA2. André Guinier avait auparavant obtenu de l'Université que ce regroupement puisse voir le jour.

On peut déjà dire qu'à cette époque, André Guinier était très connu de la communauté des cristallographes mais aussi de celle des métallurgistes et même bien au-delà. André Guinier est donc célèbre, et il va développer de nombreuses relations internationales. II ira ainsi donner de nombreux cours de radiocristallographie dans diverses universités (Université américaine du Caire, Harvard, Université de l'llinois, de Buenos-Aires...). II développe, avec ses élèves qui viennent de différents pays, une véritable école de radiocristallographie.

De nombreuses responsabilités lui seront naturellement confiées au cours de sa carrière. II devient vice-président de la Société de Métallurgie (1951), vice-doyen de la Faculté des Sciences d'Orsay (1959-1962), président de la Société Française de Minéralogie et Cristallographie (1960), directeur du Laboratoire de Physique des Solides d'Orsay (1966-1970), président de la Société Française de Physique (1970). Au milieu des années 1950 il a joué un rôle primordial dans l'idée de la relation entre l'enseignement et la recherche. C'est dans cet esprit qu'est venue l'idée d'un enseignement pour la recherche et le lancement des Diplomes d'Études Approfondies (DEA). En liaison notamment avec Jacques Friedel, le DEA de Physique des Solides est l'un des premiers à être mis sur pied.

Lorsqu'on regarde l'œuvre, la carrière d'André Guinier, on note de manière évidente l'intérêt actif qu'il a toujours porté au rôle majeur de l'enseignement dans notre société, sa nécessaire liaison avec la recherche et l'intérêt de faire savoir au plus grand nombre les découvertes les plus récentes. Ses cours au CNAM, dont certains deviendront télévisuels, étaient tout à fait dans cet esprit.

Les livres qu'il a écrits, «Radiocristallographie » (1945) ou celui que nous avons tous utilisé pour sa clarté et sa précision, «Théorie et technique de la radiocristallographie (1956) » témoignent de cet intérêt pour la transmission du savoir. Plus récemment, "La structure de la matière ou du ciel bleu à la matière plastique » (1980), “ Les rayons X » (1983) et le dernier réalisé avec Rémy Julien en 1987, «La matière à l'état solide » témoignent de ce goût de l'enseignement et de la volonté de décrire la physique de manière simple et précise. II avait la clarté d'esprit des gens qui ont beaucoup réfléchi et laissaient uniquement les équations nécessaires à une compréhension précise.

Le rôle qu'il a joué dans le domaine de l'éducation se retrouve aussi dans les responsabilités qu'il a assumées, par exemple au sein de l'Union Internationale de Cristallographie lorsqu'il présidait (1960) la «Commission de l'enseignement » de cette organisation. II acceptait volontiers d'aider dans ce domaine même ces dernières années. C'est ainsi qu'il était encore tout récemment président du Conseil d'administration de l'Institut de Cinématographie Scientifique fondé par Jean Painlevé et actuellement dirigé par Alexis Martinet. II aimait également participer à la vie de notre société, la SF2M. Pendant de très nombreuses années, il a tenu à participer aux Journées d'Automne, et était toujours très au fait de l'évolution des matériaux et des techniques d'étude.

Il est aussi très intéressant de noter qu'André Guinier a participé activement à de nombreux congrès du début de la microscopie électronique. II voyait dans cette technique une méthode très complémentaire de l'approche des rayons X pour l'étude des matériaux. Le premier congrès international de microscopie électronique, qui s'est tenu en 1950 à Paris, garde la trace de ses interventions, comme les nombreuses réunions qui étaient organisées en France dans ce domaine par des universitaires ou des industriels.

Son humour par petites touches qui résumaient en quelques mots une situation bien définie, et dont tous ceux qui ont discuté avec lui gardent un souvenir précis et nostalgique, était proverbial. II m'avait donné une diapositive de son premier diagramme de diffusion à petit angle dont il a tiré l'information sur la structure des amas qui portent son nom. Je lui avais ensuite présenté un cliché de microscopie à haute résolution sur le même type d'alliage aluminium-cuivre où l'on voit la projection de colonnes atomiques révélant l'épaisseur atomique d'une zone GP1. II m'avait alors dit avec un petit sourire, "vous, on est bien obligé de vous croire, mais de mon temps ... ».

Membre de l'Institut, titulaire de nombreux prix, il a fait école et ses élèves ont su faire bénéficier la communauté, au travers de l'utilisation de la diffusion des rayons $X$ en laboratoire ou en utilisant les sources synchrotrons, de certaines astuces du pionnier qu'a été André Guinier. En visitant récemment la source de Campinas, les brésiliens étaient fiers de me montrer une chambre de Guinier qu'ils utilisent. Cette mémoire scientifique est bien la marque d'un très grand talent.

\section{Bernard JOUFFREY}

